\title{
Perfil do profissional de nível superior nas equipes da Estratégia Saúde da Família em Montes Claros, Minas Gerais, Brasil
}

\author{
Professional profile of healthcare providers holding university degree in Family Health Strategy teams in \\ Montes Claros, Minas Gerais, Brazil
}

\author{
Perfil de los profesionales con nivel universitario de los equipos de la Estrategia de Salud Familiar \\ en Montes Claros, Minas Gerais, Brasil
}

Simone de Melo Costa. Universidade Estadual de Montes Claros (UNIMONTES).smelocosta@gmail.com (Autora correspondente)

Maria Clara Martins Prado. Universidade Estadual de Montes Claros (UNIMONTES). claraprado@ig.com.br

Thais Nunes Andrade. Universidade Estadual de Montes Claros (UNIMONTES). tata_nunes31@hotmail.com

Eva Patrícia Pereira Araújo. Secretaria Municipal de Saúde de Montes Claros (MG). evapat@ig.com.br

Waldir de Souza e Silva Junior. Secretaria Municipal de Saúde de Montes Claros (MG). junior-odonto@hotmail.com

Zenilton Charles Gomes Filho. Secretaria Municipal de Saúde de Montes Claros (MG). zeniltonfilho@hotmail.com

Carlos Alberto Quintão Rodrigues. Universidade Estadual de Montes Claros (UNIMONTES). carlosquintao@hotmail.com

\section{Resumo}

Objetivo: o presente trabalho teve por objetivo conhecer o perfil do profissional de nível superior que integra as equipes da Estratégia Saúde da Família (ESF) no município de Montes Claros, norte de Minas Gerais, Brasil. Método: trata-se de estudo quantitativo, transversal e de caráter censitário. A coleta de dados deu-se por meio de um questionário semiestruturado e autoaplicado. Resultados: participaram 95 profissionais entre enfermeiros, médicos e cirurgiões dentistas, o que correspondeu a $83,33 \%$ dos profissionais de nível superior da ESF. A maioria era do sexo feminino $(80,0 \%)$, pós-graduado (85,3\%), que se sentia preparado para o setor público de saúde $(98,9 \%)$, com participação em atividades de educação permanente $(97,9 \%)$ e com atuação exclusiva no setor público $(77,7 \%)$. Conclusão: a qualificação profissional e a dedicação exclusiva ao sistema público de saúde revelaram características de perfil profissional importantes e condizentes com as exigências do trabalho junto à Estratégia Saúde da Família.

\section{Abstract}

Objective: In this article, we aimed to understand the professional profile of healthcare providers holding university degree in Family Health Strategy (FHS) in the municipality of Montes Claros, northern Minas Gerais state, Brazil. Method: It is a cross-sectional analysis through semi-structured questionnaires self-applied to all university degree professionals working within FHS in the city. Results: Ninety-five professionals took part in the research including nurses, doctors and dentists, corresponding to $83.3 \%$ of the professionals with higher education degree working in FHS. Most professionals were female $(80 \%)$, had done some graduate study (85.3\%), regarded themselves prepared to work at the public health sector $(98.9 \%)$, had taken part in continuing education activities (97.9\%) and worked exclusively in the public sector (77.7\%). Conclusion: Professional qualification and exclusive availability to the public health system showed important professional profile characteristics that are in line with the requirements for working with Family Health Strategy.

\section{Resumen}

Objetivo: este estudio buscó conocer el perfil profesional de aquellos integrantes del equipo de la Estrategia de Salud Familiar (ESF) que tuvieran Grado Universitario en la ciudad de Montes Claros, en el norte del Estado de Minas Gerais, Brasil. Método: Análisis transversal a través de un cuestionario semiestructurado y autoaplicado entre los profesionales de nivel superior integrantes de la ESF de la ciudad. Resultados: 95 profesionales participaron, entre enfermeras, médicos y dentistas, que representaron el $83,3 \%$ de los profesionales de nivel superior de la ESF. La mayoría eran mujeres (80\%), con posgrado (85,3\%), se sentían listos para trabajar en Salud Pública (98,9\%), habían participado en actividades de educación continua $(97,9 \%)$ y trabajaban exclusivamente en el sector público $(77,7 \%)$. Conclusión: la cualificación profesional y dedicación exclusiva al sistema de salud pública revelaran características importantes del perfil profesional, en concordancia con las exigencias del trabajo en la Estrategia Salud Familiar.

Palavras-chave:

Atenção Primária à Saúde Saúde da Família Recursos Humanos

\section{Keywords:}

\section{Palabras clave:} Atención Primaria de Salud Salud de la Familia Recursos Humanos 


\section{Introdução}

O Programa Saúde da Família (PSF) foi criado em 1994, pelo Ministério da Saúde, com o objetivo de promover a reorientação do modelo assistencial à saúde até então vigente no Brasil. Trata-se de uma política nacional instituída a partir de um projeto dinamizador do Sistema Único de Saúde (SUS) que incorpora e reafirma os princípios da universalidade, da integralidade, da equidade, do controle social, da descentralização político-administrativa, da hierarquização e da regionalização, estabelecidos na Lei Orgânica de Saúde, instituída em 1990¹. O PSF foi oficialmente transformado em Estratégia Saúde da Família (ESF) em 2006, a fim de ordenar e consolidar a Atenção Primária à Saúde (APS) no Brasil.

A ESF foi implantada nos municípios brasileiros a partir de decisões políticas direcionadas para o bem comunitário e respaldadas na reorganização do sistema de saúde nos municípios. Essa reorganização visa uma nova relação interpessoal de confiança, respeito e atenção entre comunidade e profissionais de saúde. Em adiçáo, a ESF se integra aos serviços de saúde municipal e regional, caracterizando-se como porta de entrada na rede SUS municipal ${ }^{2}$.

Cada equipe preconizada pela ESF é composta por um médico de família, um enfermeiro, um auxiliar de enfermagem e seis agentes comunitários de saúde. Quando ampliada, conta ainda com um cirurgião dentista, um auxiliar de saúde bucal e um técnico em saúde bucal2 ${ }^{2}$.

Os profissionais da APS devem ter qualificação e perfil diferenciados e serem capazes de desenvolver e organizar açôes que enfatizem a relação entre a equipe, a comunidade e os diversos setores envolvidos na promoção da saúde ${ }^{3}$. Nesse contexto, a educação permanente propóe estratégias contextualizadas e participativas, orientadas para a transformaçáo de práticas, enquanto a educaçáo continuada valoriza a ciência como fonte de conhecimento técnico-científico, mesmo que desarticulada da gestão e do controle social ${ }^{4}$. A qualificação do profissional que integra a Saúde da Família por meio da educação permanente e/ou continuada é importante, pois o desafio da ESF é o de promover uma corresponsabilidade com os usuários dos serviços de saúde e com a comunidade, proporcionando uma maior resolubilidade da atenção.

A compreensão da ESF como reorientação do modelo assistencial significa também conhecer os atores sociais envolvidos nas equipes que integram a proposta de saúde da família. Nesse contexto, o objetivo deste trabalho foi identificar o perfil do profissional de nível superior da ESF de Montes Claros, Minas Gerais, Brasil.

\section{Métodos}

A pesquisa foi aprovada pelo Comitê de Ética em Pesquisa da Universidade Estadual de Montes Claros, Unimontes, parecer no 1966/2010, em respeito à Resoluçáo 196/965 e aos princípios éticos contidos na Declaraçáo de Helsinki, tendo sido obtidos Termos de Consentimento Livre e Esclarecido de todos os sujeitos pesquisados. Este trabalho faz parte de um estudo maior conduzido no âmbito do Programa de Educação para o Trabalho, PET-Saúde proposto pelos Ministérios da Educação e da Saúde, com período de vigência 2010-2011. O PET-Saúde foi destinado a viabilizar o aperfeiçoamento e a especialização em serviço, a iniciação ao trabalho, promovendo estágios e vivências dirigidos a acadêmicos e profissionais da saúde, conforme as necessidades do SUS 6 .

Trata-se de estudo transversal, quantitativo e de caráter censitário. A proposta foi desenvolvida junto aos profissionais de saúde de nível superior vinculados à Saúde da Família, Estratégia Saúde da Família e Estratégia de Agentes Comunitários de Saúde (EACS) da zona urbana de Montes Claros, Minas Gerais, Brasil, em 2010. O estudo foi conduzido em 63 ESF/ EACS, que representam a totalidade de equipes no município cenário do estudo, no período da coleta de dados, totalizando 114 profissionais de nível superior (50 da enfermagem, 27 da medicina e 37 da odontologia). Ressalta-se que, na ESF, a equipe de profissionais de nível superior é formada por enfermeiros, médicos e cirurgióes-dentistas, enquanto na EACS participa apenas a categoria enfermagem.

Utilizou-se como instrumento de coleta de dados o questionário semiestruturado e autoaplicado em visita dos pesquisadores às Unidades de Saúde no âmbito da ESF. O questionário foi elaborado para atender os objetivos do estudo, a partir da pesquisa bibliográfica sobre a temática. Para o delineamento do perfil profissional foram incorporadas no questionário variáveis demográficas de formação profissional, de educação permanente e questôes que abordaram o trabalho junto ao SUS. Foi realizado um estudo piloto para testar e adequar o instrumento de coleta de dados. Nessa etapa participaram 
dez profissionais vinculados à ESF, entre médicos, cirurgióes-dentistas e enfermeiros, sendo que esses sujeitos não foram incluídos no estudo principal.

O banco de dados foi construído no Programa SPSS versão $18.0^{\circ}$. Para a análise estatística foram utilizadas medidas de tendência central como: média aritmética, desvio padrão, valores mínimos e máximos e quartis, bem como o cálculo de proporçôes. A associação entre as variáveis foi feita por meio do teste estatístico Qui-quadrado de Pearson. Para a comparação entre as médias dos diferentes grupos de formação profissional foram adotados os testes $t$ de Student e Anova, corrigidos pela técnica de Bonferroni quando indicado. Os testes consideraram nível de significância p<0,05 e intervalo de confiança 95\%.

\section{Resultados}

Participaram do estudo 95 profissionais de saúde de nível superior - enfermeiros, médicos e cirurgióes-dentistas - atuantes na Saúde da Família, da zona urbana de Montes Claros, Minas Gerais.

Uma grande parcela $(47,4 \%)$ dos profissionais foi de enfermeiros. A grande maioria dos entrevistados foi do sexo feminino $(80,0 \%)$. A idade dos profissionais variou de 23 a 55 anos, sendo a média igual a 29,49 anos $( \pm 6,01)$ e a mediana foi 28 anos. O percentil 75 foi igual a 31 anos de idade. A maioria dos profissionais $(79,3 \%)$ tinha pós-graduaçấo concluída e 6,9\% estavam cursando pós-graduação. As características do perfil e do trabalho junto à Saúde da Família estão demonstradas na Tabela 1.

A associação entre a categoria profissional e o sexo dos entrevistados demonstrou que, do total de 76 mulheres, $51,3 \%$ eram enfermeiras, 32,9\%, cirurgiãs-dentistas e 15,8\%, médicas. Apesar de a maioria das mulheres ser da categoria enfermagem, não foi constatada diferença estatisticamente significativa entre o sexo e a categoria profissional $(\mathrm{p}=0,10)$.

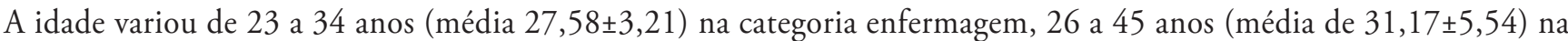
medicina e 23 a 55 anos (média 31,78 $\pm 8,70$ ) na odontologia. Houve diferença estatisticamente significante apenas entre as médias dos profissionais da enfermagem e da odontologia $(\mathrm{p}=0,02)$.

Com relação à comparação das médias de idade entre homens e mulheres foi verificada uma média de idade maior entre os homens $(32,11 \pm 8,60)$ quando comparada à média de idade das mulheres $(28,64 \pm 4,82)$, com diferença estatística $(\mathrm{p}=0,03)$.

Tabela 1. Características dos profissionais de saúde (n=95). Saúde da Família, Montes Claros, MG, Brasil, 2010.

\begin{tabular}{|c|c|c|}
\hline Variável & $\mathrm{n}$ & $\%$ \\
\hline \multicolumn{3}{|l|}{ Categoria profissional } \\
\hline Enfermagem & 45 & 47,5 \\
\hline Odontologia & 31 & 32,5 \\
\hline Medicina & 19 & 20,0 \\
\hline \multicolumn{3}{|l|}{ Sexo } \\
\hline Feminino & 76 & 80,0 \\
\hline Masculino & 19 & 20,0 \\
\hline \multicolumn{3}{|l|}{ Ter pós-graduação } \\
\hline $\operatorname{Sim}$ & 81 & 85,3 \\
\hline Não & 14 & 14,7 \\
\hline \multicolumn{3}{|l|}{ Sentir-se preparado para o SUS } \\
\hline Sim & 94 & 98,9 \\
\hline Não & 01 & 01,1 \\
\hline \multicolumn{3}{|l|}{ Conhecer o Plano Diretor da APS } \\
\hline $\operatorname{Sim}$ & 89 & 93,7 \\
\hline Não & 06 & 06,3 \\
\hline \multicolumn{3}{|l|}{ Ter participado das 10 oficinas do Plano Diretor da APS } \\
\hline $\operatorname{Sim}$ & 34 & 35,8 \\
\hline Não & 61 & 64,2 \\
\hline \multicolumn{3}{|l|}{ Trabalhar exclusivamente no SUS } \\
\hline $\operatorname{Sim}$ & 73 & 77,7 \\
\hline Não & 21 & 22,3 \\
\hline
\end{tabular}


No que diz respeito à idade e à condição de ter pós-graduação, verificou-se que a média de idade dos pós-graduados era maior do que a daqueles sem pós-graduação, 30,14( $\pm 6,48)$ e $26,79( \pm 3,02)$, respectivamente, sem no entanto haver diferença significativa $(\mathrm{p}=0,06)$.

Associando-se a condição de possuir pós-graduação concluída e a categoria profissional verificou-se que entre os pós-graduados a categoria enfermagem representou 51,9\%, a odontologia, 29,1\% e a medicina, 19,0\%, sem diferença estatística entre os grupos $(\mathrm{p}=0,13)$. As mulheres compuseram um percentual de $83,5 \%$ dos pós-graduados $(\mathrm{p}=0,05)$. Entre os pós-graduados, $47,1 \%$ cursaram a residência multiprofissional em saúde da família.

Entre os entrevistados, a quase totalidade $(98,9 \%)$ respondeu sentir-se preparada para atuar no SUS, sendo que $97,9 \%$ dos profissionais informaram ter participado de algum treinamento em serviço. Quanto ao quesito conhecer a proposta de capacitação de recursos humanos elaborada pelo governo do Estado de Minas Gerais conhecida como Plano Diretor de Atenção Primária à Saúde (PDAPS), a grande maioria (93,7\%) afirmou conhecê-la. No entanto, das 10 oficinas propostas no PDAPS, menos profissionais $(35,8 \%)$ informaram ter participado de todas elas, $48,4 \%$ participaram de algumas oficinas e $15,8 \%$ não participaram de nenhuma.

Entre os 34 profissionais que participaram de todas as oficinas do PDAPS foi constatado que com relação à categoria profissional a maioria dos participantes (52,9\%) eram cirurgióes-dentistas, $26,5 \%$ eram enfermeiros e 20,6\%, médicos $(\mathrm{p}<0,01)$. Ainda com relação aos participantes do PDAPS em sua totalidade, verificou-se que as mulheres compuseram o maior percentual de participação $(70,6 \%)$, apesar de não haver diferença significativa entre os sexos $(\mathrm{p}=0,09)$.

A maioria dos profissionais $(77,7 \%)$ atuava exclusivamente no serviço público. Entre esses profissionais que atuavam somente no serviço público a maioria era do sexo feminino $(\mathrm{p}=0,17)$, formados em enfermagem $(\mathrm{p}=0,60)$ e tinha pós-graduação $(\mathrm{p}=0,78)$. Observou-se que o trabalho exclusivo no setor público de saúde não foi associado à participação em todas as 10 oficinas do PDAPS $(\mathrm{p}=0,41)$.

\section{Discussão}

Participaram do estudo 95 profissionais de saúde de nível superior - enfermeiros, médicos e cirurgióes-dentistas - atuantes na Saúde da Família de Montes Claros, Minas Gerais, Brasil, o que equivale a 83,3\% dos profissionais atuantes em 2010. $\mathrm{O}$ fato de a maioria dos profissionais serem enfermeiros era um resultado esperado uma vez que o trabalho foi executado também junto à EACS, que não incorpora as categorias médica e odontológica na equipe de trabalho.

Com relação ao aspecto da feminização encontrado entre os profissionais da ESF, o resultado é compatível com a literatura, que revelou ser a feminizaçáo das profissóes uma das tendências da área da saúde e, por conseguinte, dos profissionais das equipes de saúde da família ${ }^{7}$. O fenômeno da feminização também foi constatado ainda no período da graduação em saúde . $^{8}$ Fatores como a expansão e a elevação dos níveis de escolaridade e instrução, acrescidos da redução das taxas de fecundidade, são razóes que impulsionaram a mulher para o mercado de trabalho remunerado. Acredita-se também que as mudanças econômicas ocorridas no Brasil, ao longo de décadas passadas, propiciaram a formação de um mercado de trabalho aberto para a mulher, principalmente no setor terciário da economia, fato que permitiu o seu avanço atual para outras instâncias e setores ${ }^{7}$.

A maior presença de profissionais jovens atuando na Saúde da Família poderia ser justificada pela mudança curricular nos cursos de graduação. A formação em saúde tem enfatizado a capacitação profissional para atender as reais demandas da Atenção Primária à Saúde. Nesse sentido, programas do Ministério da Saúde e da Educação como o Programa Pró-Saúde têm contribuído para reorientar a formação em saúde. O Pró-Saúde estimula o contato do estudante com o serviço público, capacitando-o para açôes junto ao SUS ${ }^{9}$. E isso pode estimular os recém-formados a buscar oportunidade de trabalho junto ao SUS ${ }^{10}$.

A grande prevalência de profissionais que cursaram ou estão cursando uma pós-graduação demonstra uma tendência de busca de melhor qualificação por meio de cursos de especialização na área da saúde. Ademais, o Ministério da Saúde tem adotado a estratégia de favorecer a qualificação profissional no sentido de fortalecer as políticas no setor público de saúde ${ }^{11}$. Cabe ressaltar que o município cenário deste estudo propôs no período de 1997/1998 a oferta de um Programa de Residência Multiprofissional em Saúde da Família para médicos e enfermeiros. A proposta surgiu após a participação da Universidade Estadual de Montes Claros (Unimontes) no Five Weekend Programme. A inclusão da Unimontes no programa 
foi uma estratégia da Secretaria de Saúde do Estado de Minas Gerais em parceria com a Universidade de Toronto, Canadá, com o objetivo de oferecer um curso capaz de qualificar médicos e enfermeiros da ESF de diferentes cidades mineiras. O primeiro curso de especialização na modalidade Residência em Saúde da Família teve início em $1999^{12}$. Esse fato histórico pode ter contribuído, em parte, para a condição apresentada pela maioria dos profissionais deste estudo: ter especialização. Isso porque a maior parte dos entrevistados tinha especialização na área saúde da família.

No presente estudo, a grande maioria dos entrevistados respondeu sentir-se preparado para atuar no SUS, resultado importante para a Estratégia Saúde da Família. Por outro lado, o "sentir-se preparado" é um aspecto subjetivo, o que não garante a qualidade do serviço prestado.

A qualidade do serviço em saúde pode ser influenciada por diferentes fatores. Entre eles podem-se destacar a presença ou ausência de infraestrutura adequada, de uma equipe multiprofissional e capacitada e os aspectos administrativos ${ }^{13}$. Nesse sentido, o sentir-se preparado para o SUS é importante mas não suficiente para garantir a qualidade dos serviços em saúde.

O fato de este estudo ter demonstrado que a maioria dos trabalhadores sentia-se capacitada para o serviço público contraria os resultados encontrados na literatura ${ }^{11,14,15}$.

Em outros estudos foi constatado que o perfil dos profissionais formados não era adequado para atuar na perspectiva de atenção integral à saúde. Além disso, estudos constataram que os profissionais, na maioria das vezes, não realizavam satisfatoriamente açôes de promoção, proteção, prevenção e atenção precoce nos serviços de saúde públicos ${ }^{11,14,15}$.

O treinamento em serviço foi indicado pela grande maioria dos entrevistados, que informou conhecer o Plano Diretor de Atenção Primária à Saúde (PDAPS). O Plano Diretor consistiu em diretrizes propostas pela Secretaria do Estado de Saúde de Minas Gerais (SES/MG) para o enfrentamento dos problemas que impedem a efetiva função da APS na rede de atenção SUS ${ }^{16}$. As oficinas do PDAPS aconteceram em datas pré-agendadas, em dias diferentes, e contaram com o apoio dos gestores para a participação efetiva dos funcionários da ESF nas atividades. Apesar da quase totalidade (93,7\%) conhecer o PDAPS, apenas 35,8\% dos entrevistados participaram das dez oficinas.

A constatação da não participação efetiva em atividades de educação permanente também foi verificada em outro estudo ${ }^{17}$. A baixa adesão, por parte dos profissionais de saúde, às atividades de educação permanente promovidas pelos órgãos públicos está em desacordo com as propostas do SUS. Isso porque a atuação na APS requer uma capacitação permanente e continuada dos profissionais.

Entre os participantes de todas as oficinas do PDAPS, o fato de a maior participaçáo referir-se ao grupo da odontologia é um resultado relevante. Esse resultado demonstra, de certo modo, o interesse dos cirurgióes-dentistas pelas questôes da Saúde da Família, apesar da inserção da odontologia na ESF ter ocorrido somente após seis anos da implantação da estratégia.

As oficinas do PDAPS podem ser caracterizadas como atividades de educação permanente, a qual, por sua vez, se fundamenta na concepção de educação como transformação e aprendizagem significativa. A educação permanente está centrada no exercício cotidiano do processo de trabalho, valorizando o trabalho como fonte de conhecimento e articulando atenção à saúde, gestão e controle social. Ela também é orientada para a transformação de práticas ${ }^{4}$. A estratégia metodológica adotada na condução das oficinas do PDAPS para os trabalhadores da ESF é compatível com educação permanente em serviço.

O fato de a maioria dos profissionais informarem atuação exclusivamente no serviço público foi um resultado esperado, independentemente de sexo, categoria profissional e de ter ou não pós-graduação. Isso porque, em 28 de março de 2006, o Ministério da Saúde aprovou a Portaria de no 698 (capítulo II, parágrafo 2.1, inciso IV) que definiu a carga horária de 40 horas semanais para todos os profissionais nas equipes de saúde da família e saúde bucal ${ }^{18}$.

Para os poucos profissionais que afirmaram também trabalhar no setor privado, tem-se por hipótese que eles buscam uma complementação da renda mensal. É provável que a atividade junto ao setor privado se dê através da execução de plantôes por parte dos profissionais da medicina e da enfermagem, determinando uma dupla jornada de trabalho após o cumprimento das oito horas diárias na ESF.

Em 2011, a Portaria no 2.027 alterou a determinação da carga horária de oito horas diárias, estabelecendo diferentes configuraçôes de jornada de trabalho para os profissionais médicos da ESF, permitindo jornadas também de 20 ou 30 horas semanais. Essa mudança poderá interferir na forma de atuação dos profissionais no setor público ${ }^{19}$ visto que o trabalho de profissionais da área da saúde em turnos favorece múltiplos vínculos empregatícios. No entanto, não se pode desconsiderar que o excesso de trabalho pode determinar riscos e prejuízo para a qualidade da assistência à saúde e para a saúde do trabalhador ${ }^{20}$.

Outra hipótese para a necessidade de atuação concomitante no serviço privado pode estar associada também aos aspectos desfavoráveis no setor público, como o vínculo precário na contratação do profissional' . A proliferação de contratos informais 
de trabalho nos diferentes setores do mercado nacional atingiu também a saúde pública. Entre as repercussôes dessa realidade estão a privação de direitos garantidos pela lei trabalhista brasileira, como o direito a férias, ao Fundo de Garantia por Tempo de Serviço (FGTS), licenças, $13^{\circ}$ salário e contribuiçôes previdenciárias para a garantia da aposentadoria. Devido às falhas no cumprimento dos direitos trabalhistas, no Brasil, os profissionais permanecem suscetíveis à instabilidade políticopartidária dos municípios ${ }^{3}$.

Nesse sentido, o Ministério da Saúde desenvolveu uma política de valorização do trabalhador denominada DesprecarizaSUS. Essa política definiu o trabalho precário pela ausência de direitos trabalhistas e previdenciários legais relacionada às frequentes terceirizaçóes e à inexistência ou irregularidade de contratos ${ }^{20}$.

Esses aspectos desfavoráveis de trabalho dos profissionais atuantes no setor público são reflexos da instabilidade profissional, uma vez que a legislação do SUS é insuficiente para garantir a segurança do trabalhador. As portarias nacionais vigentes, que tratam da organizaçáo da ESF delegam às prefeituras municipais a responsabilidade de contratação dos profissionais, não exigindo a realização de concurso público? .

Nesse sentido, estudo realizado com profissionais da atenção primária à saúde nas regiōes Sul e Nordeste do Brasil demonstrou que menos da metade de 4.749 funcionários ingressaram ao SUS por concurso público. Os resultados revelaram que a precarização do trabalho foi ampliada a partir da expansão da saúde da família, de forma mais agravante na região Nordeste, onde a estratégia foi adotada precoce e intensamente ${ }^{21}$. Se a contratação ocorre por indicação política, a classe dos trabalhadores fica à mercê dos gestores municipais e, desse modo, essa condição pode refletir em intimidação dos profissionais para a requisição de direitos enquanto trabalhadores da saúde.

\section{Conclusões}

O quadro profissional de nível superior que atua na Estratégia Saúde da Família de Montes Claros, Minas Gerais, é formado principalmente por pós-graduados, com autopercepção de preparo para as atividades requeridas junto ao SUS e majoritariamente com dedicação exclusiva ao setor público.

A qualificação profissional e a dedicação exclusiva ao sistema público de saúde revelam características de perfil profissional importantes e condizentes com as exigências do trabalho junto à Estratégia Saúde da Família.

\section{Agradecimentos}

Ao Programa de Educação para o Trabalho em Saúde (PET-Saúde) 2010-2011 pelo incentivo à investigação científica integrada com profissionais do serviço público de saúde, acadêmicos e professores universitários.

\section{Referências}

1. Martines WRV, Chaves EC. Vulnerabilidade e sofrimento no trabalho do agente comunitário de saúde no Programa de Saúde da Família. Rev. Esc. Enferm USP. 2007; 41(3): 426-33. [citado 2012 Mar 20]. http://dx.doi.org/10.1590/S0080-62342007000300012

2. Brasil. Ministério da Saúde. Secretaria de Políticas Públicas. Guia prático do Programa de Saúde da Família. Brasília: Ministério da Saúde; 2001. [Internet]. Disponível em: http://dtr2002.saude.gov.br/caadab/arquivos\%5Cguia_psf1.pdf

3. Cotta RMM, Schott M, Azeredo CM, Franceschini SCC, Priore SE, Dias G. Organização do trabalho e perfil dos profissionais do Programa Saúde da Família: um desafio na reestruturação da atenção básica em saúde. Epidemiol Serv Saúde 2006; 15(3):7-18. http://dx.doi.org/10.5123/S167949742006000300002

4. Peduzzi M, Guerra DAD, Braga CP, Lucena FS, Silva JAM. Atividades educativas de trabalhadores na atenção primária: concepções de educação permanente e de educação continuada em saúde presentes no cotidiano de Unidades Básicas de Saúde em São Paulo. Interface. Comunic., Saúde, Educ. 2009; 13(30): 121-134. [citado 2012 fev 25]. http://dx.doi.org/10.1590/S1414-32832009000300011

5. Brasil. Ministério da Saúde. Conselho Nacional de Saúde. Resolução 196. Aprova as diretrizes e normas regulamentadoras de pesquisas envolvendo seres humanos. Brasília: Conselho Nacional de Saúde; 1996. 
6. Brasil. Ministério da Saúde. Portaria Interministerial n 1 802, de 26 de agosto de 2008. Institui o Programa Nacional de Educação pelo Trabalho para a Saúde - PET Saúde. Diário Oficial da República Federativa do Brasil, Brasília; 27 ago. 2008. Seção 1, p. 27.

7. Pinto ESG, Menezes RMP, Villa TCS. Situação de trabalho dos profissionais da Estratégia Saúde da Família em Ceará-Mirim. Rev Esc Enferm USP. 2010; 44(3): 657-64. [citado 2012 mar 23]. http://dx.doi.org/10.1590/S0080-62342010000300015

8. Costa SM, Durães SJA, Abreu MHNG. Feminização do curso de odontologia da Universidade Estadual de Montes Claros [Internet]. Ciênc. saúde coletiva 2010; 15(supp.1): 1865-73. [citado 2012 fev 25]. http://dx.doi.org/10.1590/S1413-81232010000700100

9. Brasil. Ministério da Saúde. Pró-saúde: programa nacional de reorientação da formação profissional em saúde. Brasília: Ministério da Saúde; 2005.

10. Medeiros CRG, Junqueira AGW, Schwingel G, Carreno I, Jungles LAP, Saldanha OMFL. A rotatividade de enfermeiros e médicos: um impasse na implementação da Estratégia de Saúde da Família. Ciênc Saúde Coletiva. 2010; 15(suppl.1): 1521-31. [citado 2012 fev 25]. http://dx.doi.org/10.1590/ S1413-81232010000700064

11. Gil CRR. Formação de recursos humanos em saúde da família: paradoxos e perspectiva. Cad Saúde Pública. 2005; 21(2): 490-8. [citado 2012 fev 25]. http://dx.doi.org/10.1590/S0102-311X2005000200015

12. Brasil. Ministério da Saúde. Secretaria de Gestão do Trabalho e da Educação na Saúde. Departamento de Gestão da Educação na Saúde. Residência multiprofissional em saúde: experiências, avanços e desafios. Brasília: Ministério da Saúde; 2006.

13. Loch-Neckel G, Seemann G, Eidt HB, Rabuske MM, Crepaldi MA. Desafios para a ação interdisciplinar na atenção básica: implicações relativas à composição das equipes de saúde da família. Ciênc Saúde Coletiva. 2009; 14 (suppl.1): 1463-72. [citado 2012 fev 25]. http://dx.doi.org/10.1590/ S1413-81232009000800019

14. Romano VF. A busca de uma identidade para o médico de família. Physis. 2008; 18(1): 13-25. http://dx.doi.org/10.1590/S0103-73312008000100002

15. Moretti-Pires RO, Bueno SMV. Freire e formação para o Sistema Único de Saúde: o enfermeiro, o médico e o odontólogo. Acta Paul Enferm. 2009; 22(4): 439-44. http://dx.doi.org/10.1590/S0103-21002009000400015

16. Costa SM, Dias OV, Oliveira GS, Nascimento JE, Fonseca VIV, Silva LMC. Plano Diretor da Atenção Primária à Saúde: Perfil e Atuação Profissional dos Facilitadores de Microrregiões do Norte de Minas Gerais. Rev Pró-univerSUS 2011; 2(1): 75-82.

17. Cotrim-Guimarães IMA. Programa de educação permanente e continuada da equipe de enfermagem da clínica médica do Hospital Universitário Clemente de Faria: análise e proposições. [Dissertação]. Rio de Janeiro: Escola Nacional de Saúde Pública Sergio Arouca; 2009. Disponível em: http://bvssp.icict.fiocruz.br/pdf/25620.pdf.

18. Brasil. Ministério da Saúde. Portaria nº 648 de 28 de março de 2006. Aprova a Política Nacional de Atenção Básica estabelecendo a revisão das diretrizes e normas para a organização da Atenção básica para o Programa Saúde da Família (PSF) e Programa Agentes Comunitários de Saúde (PACS). Diário Oficial da República Federativa do Brasil, Brasília; 2006.

19. Brasil. Ministério da Saúde. Portaria n 2.027, de 25 de agosto de 2011. Altera a Portaria nº 648/GM/MS, de 28 de março de 2006 , na parte que dispõe sobre a carga horária dos profissionais médicos que compõem as Equipes de Saúde da Família (ESF) e na parte que dispõe sobre a suspensão do Piso de Atenção Básica (PAB Variável) [internet]. Diário Oficial da República Federativa do Brasil, Brasília; 2011. Disponível em: http://bvsms. saude.gov.br/bvs/saudelegis/gm/2011/prt2027_25_08_2011.html

20. Murofuse NT, Rizzotto MLF, Muzzolon ABF, Nicola AL. Diagnóstico da situação dos trabalhadores em saúde e o processo de formação no polo regional de educação permanente em saúde. Rev Latino-Am Enferm. 2009; 17(3): 314-20. [citado 2012 mar 12]. http://dx.doi.org/10.1590/S010411692009000300006

21. Tomasi E, Facchini LA, Piccini RX, Thumé E, Silveira DS, Siqueira FV, et al. Perfil sócio-demográfico e epidemiológico dos trabalhadores da atenção básica à saúde nas regiões Sul e Nordeste do Brasil. Cad Saúde Pública. 2008; 24 (suppl.1):193-201. [citado 2012 abr 06]. http://dx.doi.org/10.1590/ S0102-311X2008001300023 